\title{
Steuerung der Ärzte durch ein neues nationales Qualitätszentrum: Vorsicht ist geboten
}

\author{
Der Bundesrat will ein Qualitätszentrum gründen. Die Abschaffung der Schluss- \\ expertise bei der Hörgeräteanpassung durch das Bundesamt für Sozialversiche- \\ rungen zeigt, dass es nicht um die Qualitätssicherung geht, sondern um systema- \\ tische Kontrolle von Ärzten und Spitälern. Es gilt, die Vernehmlassung wahrzuneh- \\ men und sich kritisch gegen das Gesetz auszusprechen.
}

\section{Thomas Linder}

Chefarzt Klinik für Hals-, Nasen-, Ohren-, Hals- und Gesichtschirurgie, Luzerner Kantonsspital

Ich danke Prof. Dr. med. Marcus M. Maassen (Präsident PULSUS) für die kritische Durchsicht des Manuskripts.

Keine Interessenverbindungen, die den Inhalt dieses Manuskripts beeinträchtigen könnten.

Korrespondenz:

Prof. Dr. med. Thomas Linder Klinik für Hals-, Nasen-, Ohren-, Hals- und Gesichtschirurgie, Luzerner Kantonsspital Spitalstrasse

CH-6000 Luzern

Tel. 0412054951

Fax 0412054995

hno[at]luks.ch
Der Bundesrat hat am 14.5.2014 das Departement des Inneren beauftragt, ein Vernehmlassungsverfahren zu einem Entwurf zum Bundesgesetz über das Zentrum für Qualität in der obligatorischen Krankenpflegeversicherung durchzuführen.

Mit dem vorgeschlagenen Gesetz sollen die Verbesserung der Qualität der Leistungen, eine Erhöhung der Patientensicherheit sowie eine Kostendämpfung in der Krankenversicherung erreicht werden. Wer könnte sich bei diesen ehrenhaften Zielvorgaben schon dagegen wehren? Zur Finanzierung der Aufgaben sollen die Versicherer für jede nach KVG versicherte Person mit Ausnahme der Kinder zu Lasten der obligatorischen Krankenpflegeversicherung einen jährlichen Beitrag an das geplante Zentrum entrichten. Ferner würde der Bund dem geplanten Qualitätszentrum jährliche Beiträge zur Abgeltung der Aufgaben entrichten (Art. 14 des Bundesgesetzes ist über das Zentrum für Qualität in der obligatorischen Krankenpflegeversicherung).

Der Bundesrat schätzt die Kosten des mit 30 Stellen dotierten Institutes auf 32 Millionen Franken pro Jahr (NZZ vom 11.1.2014). Von diesen Kosten werden 22 Millionen Franken für das Segment Qualitätssicherung und Patientensicherheit benötigt. Dies würde durch eine jährliche Prämienerhöhung von 3.50 Franken pro versicherter Person entsprechen, denn der Bundesrat beabsichtigt, das neu zu gründende Zentrum über Beiträge der Versicherten (Kinder ausgenommen) zu finanzieren. Dies entspricht also einer staatlich verordneten Prämienerhöhung, denn die Versicherungen werden diese Kosten an die Versicherten weiterleiten. Zusätzlich wird pro Jahr für das Aufgabengebiet der Überprüfung medizinischer Leistungen weitere 10 Millionen Franken benötigt, die über Bundesmittel, also steuerfinanzierte Mittel, abgegolten werden.

Es entbehrt nicht einer gewissen Pikanterie, dass der Bund einerseits von den Ärzten und den Spitälern eine Kostenkontrolle einfordert, für viele Fach-

\section{Régulation des médecins}

\section{par un nouveau Centre national de la qualité}

Dans le but d'accroître la qualité et la sécurité des patients, le Conseil fédéral entend créer un Centre national pour la qualité comportant 30 places de travail pour un coût de CHF 32 millions par an. Alors que médecins et hôpitaux sont constamment tenus de contrôler leurs coûts, que le temps de travail des médecins-assistants est revu à la baisse et que le corps médical est confronté à un flot de certifications, la Confédération se targue de jouer les assureurs-qualité. La suppression par l'OFAS de l'expertise finale pour l'adaptation des appareils auditifs en 2011 montre pourtant bien que la Confédération ne se préoccupe pas tant de la qualité des prestations médicales et de la sécurité des patients, mais qu'elle recherche plutôt un contrôle systématique des médecins et des hôpitaux. La rémunération forfaitaire des appareils auditifs et la suppression du contrôle final par le médecin avant appareillage témoignent du manque de vue de la Confédération pour améliorer la qualité des prestations médicales. La Confédération veut forcer à une grande quantité de données et amplifier ainsi son impact sur le paysage hospitalier, au travers notamment de la médecine hautement spécialisée et des données des études sur la qualité. Pour toutes ces raisons, il faut saisir l'occasion de se prononcer sur cette loi en participant à la procédure de consultation d'ici au 5 septembre 2014. 


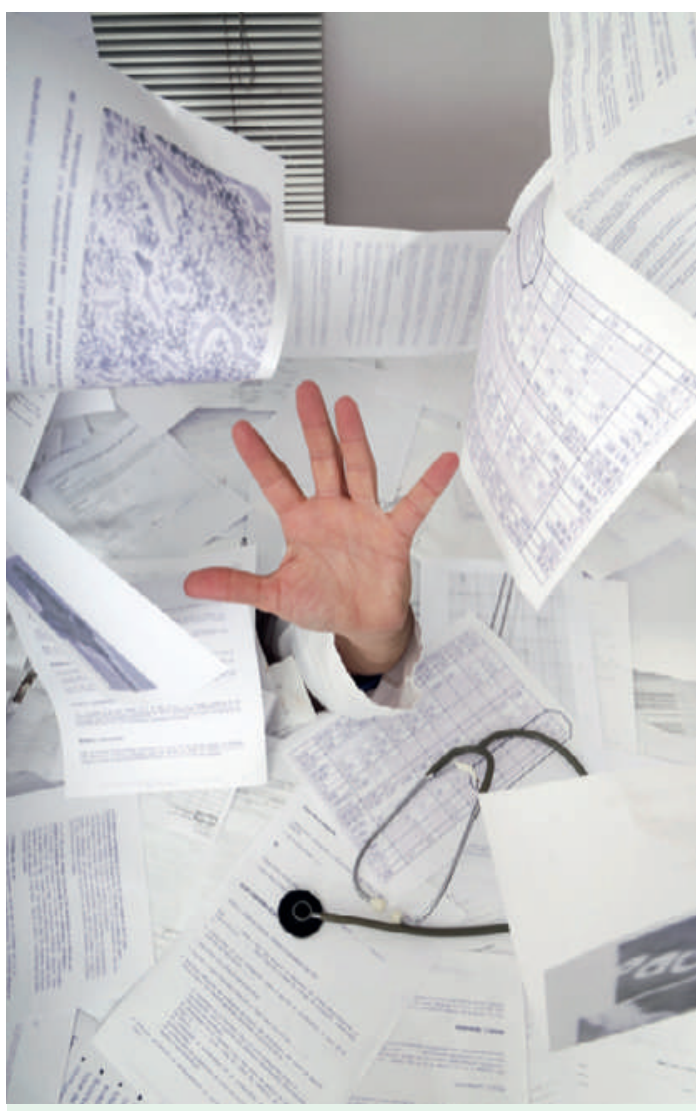

Ärzte brauchen endlich wieder mehr Zeit für Stethoskop und Skalpell, statt Checklisten, Zertifizierungen, Arbeitsgesetze und Re-Zertifizierungen umzusetzen.

disziplinen den TARMED-Tarif ohne sachgerechte und betriebswirtschaftliche Begründung senkt und andererseits - wenn es um die Beschaffung von Daten für den Bund geht - jährliche Unterhaltskosten von 32 Millionen Franken gutheisst.

Der Markt der Qualitätsverbesserer stieg in den letzten Jahren sprunghaft an. Neben der Kodierungswelle werden wir gezwungen, aufwendige perioperative Checklisten mehrfach auszufüllen, wobei eine sächliche Benefit für den Patienten verteilt sich eher in Hochglanzbroschüren als in wissenschaftlichen Beurteilungen. Damit sich der Qualitätsmarkt weiter vergrössern und sicher erhalten kann, wird selbstverständlich in festgelegten Abständen re-zertifiziert.

Daneben befindet der Bund, dass die Ärzte in Weiterbildung viel zu viel arbeiten, damit den Patienten gefährden und somit die 50-Stunden-Woche nach Arbeitsgesetz für Assistenten und bald auch Oberärzte umgesetzt werden muss. Dies also unter der Prämisse der «Verbesserung der Qualität der Leistungen». Lieber also einen ausgeruhten Anfänger am Operationstisch, mit leider wenig Erfahrung (da sich ja das Patientengut auf immer mehr Auszubildende verteilt), als einen erfahrenen Operateur mit vielen Arbeitsstunden. In einer Klammerbemerkung mag man anführen, dass für diese Qualitäts- und Zertifizierungsprozesse mit Erstellung von zahlreichen Papierordnern meist erfahrene Ärzte betraut werden, die sich sonst gerne am Operationstisch nützlich gemacht hätten.

Wenn der Bund das Ziel anstrebt, die Verbesserung der Qualität und des zweckmässigen Einsatzes der Leistungen sowie eine Förderung der Sicherheit bei der Erbringung der Leistungen durch das neu zu gründende «Zentrum für Qualität in der obligatorischen Krankenpflegeversicherung» zu realisieren, dann stellt sich insbesondere für das Fachgebiet der Hals-Nasen-Ohrenerkrankungen die Frage, warum dann in der Verordnung der Hörgeräte die Zweitexpertise abgeschafft worden ist. Zur Erinnerung: Es wurde ein qualitativ hochwertiges Versorgungssystem im internationalen Vergleich als zu teuer eingestuft und seit 1. Juli 2011 ein günstiges Pauschalsystem eingeführt [2]. Vor 2011 wurde ein Hörgerät vom ORL-Expertenarzt (also einem ORL-Arzt mit der zusätzlichen Qualifizierung) indiziert, vom zertifizierten Hörgeräteakustiker angepasst und die abgeschlossene Hörgeräteversorgung erneut vom ORLExpertenarzt mittels objektivierbarer Hörtestungen überprüft. Die IV oder AHV bezahlte die Leistungen

\section{«Nicht-Mediziner werden Qualitätsprojekte ausdenken und uns Mediziner zur zusätzlichen Datenerfassung zwingen.»}

kürzlich im NEJM dazu verfasste kritische Arbeit aus Kanada (keine Verbesserung der Patientensicherheit) kaum gelesen wird [1]. Nun dürfen sich viele grössere Spitäler zertifizieren (auch beim Autor dieser Arbeit), sei es z.B. als Tumorzentrum, Implantatzentrum, oder ihre Abläufe nach ISO-Normen bestätigen lassen. Das dabei erarbeitete Papier ist schier unermesslich, die unzähligen Arbeitsstunden können niemandem verrechnet werden und der tat- des Akustikers und das Gerät nach festgelegten Indikationsstufen und finanzierte auch die Erst- und Schlussexpertise des ORL-Arztes. Mit dem Wechsel zum Pauschalsystem wird zwar noch eine Erstexpertise gefordert, der deutlich tiefere Pauschalbetrag wird nun aber dem Patienten direkt ausbezahlt und die Schlussexpertise wurde gänzlich fallengelassen. Diese Zweitexpertise hatte jedoch nur eine Funktion: die Qualität der Hörgeräteversorgung zu kont- 
rollieren. Sie war somit ein reines Qualitätsinstrument. Das Bundesamt für Sozialversicherungen (BSV) begleitete deren Abschaffung und die Einführung des neuen Pauschalsystems mit einer eigenen, einem deutschen Institut in Auftrag gegebenen Qualitätsstudie, die sich nun - wen wundert's - als sogenannter «Forschungsbericht» lesen lässt (www. bsv.admin.ch). Während die eigene Beurteilung natürlich durchwegs positiv ausfällt, zeigt eine kürzlich in Deutschland erschienene Arbeit, dass die unkontrollierte Hörgeräteabgabe durchaus «Komplikationen» aufweisen kann [3]. Wir Ärzte hätten mit einer eigenen Qualitäts- oder eben Forschungsstudie aufwarten sollen, aber wer an den Kliniken hat neben der Umsetzung von Checklisten, Zertifizierungen, Arbeitsgesetzen und Re-Zertifizierungen denn noch Zeit, freiwillig eine Qualitätsstudie anzuregen, geschweige denn umzusetzen?

Das Beispiel der Abschaffung der Zweit- oder Schlussexpertise ist nämlich ein Paradebeispiel, das offenbart, dass es dem Bund weniger um die Verbes-
Aus ärztlicher Sicht mahne ich daher zur speziellen Vorsicht, denn mit dem neu zu gründenden Institut für Qualität wird der Weg zu mehr Planwirtschaft und mehr Staatsmedizin weiter beschleunigt. Das Gesetz ist schädlich für den freien Arztberuf und für die Spitäler und ist daher abzulehnen. Einzig erfreulich ist, dass dieses Gesetz dem fakultativen Referendum untersteht (Art. 25 des Gesetzes). Gebt uns Ärzten endlich wieder mehr Zeit für Stethoskop und Skalpell, statt Bundesstellen für zusätzliche 32 Millionen jährlich zu kreieren.

Der interessierte Leser meint vielleicht, dass ich als Autor dieser Zeilen kein Interesse an der Qualitätsmessung hätte. Hier möchte ich einwenden, dass eine meiner Forschungsaktivitäten seit 10 Jahren die Entwicklung einer Qualitätsdatenbank ist, nach dem Prinzip «warum braucht der Kliniker eine Forschungsdatenbank und der Forscher eine klinische Datenbank», und ich dabei mit der Firma innoforce mehrere Module für die ORL entwickelt und erforscht habe, die in meiner Klinik (und auch

\section{«Ich ermutige die FMH, die fmCh und die Fachgesellschaften, sich aktiv am Vernehmlassungsverfahren zu beteiligen.»}

serung der Qualität und Patientensicherheit geht, sondern vielmehr um eine systematische Kontrolle der Ärzte und Spitäler. Er lässt durch das neu zu gründende Zentrum Daten sammeln, aus denen er weitere gesundheitspolitische Entscheidungen ableitet. Nicht-Mediziner werden Qualitätsprojekte ausdenken und uns Mediziner zur zusätzlichen Datenerfassung zwingen. Per Knopfdruck liessen sich in der Zukunft mit dem «Zentrum für Qualität» Tausende Standardbriefe generieren, mit denen die Mediziner in Spital und Praxis aufgefordert werden würden, noch mehr Daten hinsichtlich ihrer Arbeitsqualität zu liefern. Unsere zunehmend von Bern regulierte Arbeitszeit werden wir für diese Qualitätsdaten einsetzen müssen, mit der Konsequenz, dass die Ausbildung am Patienten weiter schwindet. Das kann Bundesbern egal sein, da mit dem Instrument der HSM und dem vielen Datenmaterial dann nur noch wenige Spitäler für bestimmte Eingriffe zugelassen werden und sich die bisher kollegial verhaltenden Ärzte bei der Auswahl dieser Spitäler nur noch gegenseitig zerfleischen können. anderen) ohne Zwang und ohne finanzielle Unterstützung durch den Bund umgesetzt werden und deren Verwendung es meinen Ärzten erlaubt, klinische Studien zu publizieren, Vorträge an Kongressen zu halten und damit die medizinische Qualität unserer Arbeit kontinuierlich überprüft wird.

Nun, ich ermutige die $\mathrm{FMH}$, die fmCh und die Fachgesellschaften, sich aktiv am Vernehmlassungsverfahren zu beteiligen. Die Vernehmlassungsfrist endet am 5.9.2014 (Einreichungen: abteilung-leistungen[at] bag.admin.ch; z.Hd. Herrn Christian Salchli; Tel. 058 465 3153; Internet: www.bag.admin.ch).

\section{Literatur}

1 Urbach DR, Govindaranjan A, Saskin R, Wilton AS, Baxter NN. Introduction of surgical safety checklists in Ontario, Canada. N Engl J Med. 2014;370:1029-38.

2 Linder T. Hörgeräteabgabe in Seldwyla - retten, was nicht mehr zu retten ist. Schweiz Ärztezeitung. 2011;92(28/29):1127-9.

3 Löhler J, Akcicek B, Wienke A, Hoppe U. Komplikationen bei der Hörgeräteversorgung ohne HNO-Arzt. HNO. 2014;62:360-6. 